\title{
Genetic Variability and Vegetative Compatibility in Aspergillus niger Isolated from Various Food Substances in Benin City Nigeria
}

\author{
*EBOIGBE, L; OMOREGBE, MO \\ Department of Plant Biology and Biotechnology, University of Benin, PMB 1154, Benin City, Edo State, Nigeria. \\ *Corresponding Author Email: lugard.eboigbe@uniben.edu Tel.: +2348150917824
}

\begin{abstract}
In this investigation, Aspergillus niger isolated from eight food substances, have been classified based on the absence of heterokaryon formation. The size of their sporangia were differentiated, the wild and mutant strains were subjected to vegetative compatibility tests in order to group them into different vegetative compatibility groups (VCGs) which include VCG-1, VCG-2, VCG-3 and VCG-4. The strains were further tested for the possible formation of a stable heterokaryon using nit mutants generated on potato dextrose agar (PDA) containing $2.5 \%$ chlorate $\left(\mathrm{KClO}_{3}\right)$, represented as PDC. Based on the vegetative compatibility groups, nit mutants were paired on a minimal medium (MM) for complementation test. Interestingly, there was compatibility with mycelia showing anastomoses but without the formation of heterokaryon. The vegetative compatibility groups suggested four genotypes and polymorphism in the het loci. A population study for detailed genotyping is suggested in order to unravel the genetic recombination in A. niger.
\end{abstract}

DOI: https://dx.doi.org/10.4314/jasem.v24i12.23

Copyright: Copyright (C) 2020 Eboigbe and Omoregbe. This is an open access article distributed under the Creative Commons Attribution License (CCL), which permits unrestricted use, distribution, and reproduction in any medium, provided the original work is properly cited.

Dates: Received: 01 October 2020; Revised: 20 November 2020; Accepted: 16 December 2020

Keywords: Vegetative compatibility, heterokaryon, Het loci, genotype, mutant, polymorphism.

Aspergillus niger (Tiegh) is a common saprophyte that is found in the soil, feed products and stored food (Diener 1960). This filamentous fungus has a wide array of oxidative and hydrolytic enzymes involved in the breakdown of plant lignocellulose (Gautam et al., 2011) and it is known to be responsible for the spoilage of food substances. Currently, sexual stage in A. niger is not known (Frisvad et al., 2014). The genetic basis of recombination is well established in some sexual filamentous fungi but is unknown in the (presumably) asexual A. niger (Frisvad et al., 2014). This currently hinders the involvement of $A$. niger in genetic analysis. Unfortunately, this organism is very important industrially, as it is known to be involved in citric acid extraction and also in the production of cell wall hydrolyzing enzymes. A niger is seen as an industrial workhorse due to its unique integral physiological characters and better fitness for industrial fermentation (Legisa and Mattey, 2007; Papagianni, 2007; Show et al., 2015). Attempt to unravel the genetic basis of recombination in $A$. niger has met with obstacle due to lack of heterokaryon formation. This mean that mitotic recombination between genetically dissimilar isolates is blocked. Van Diepeningen et al., (1997) noted that natural isolates of black Aspergilli are highly incompatible with each other thus blocking the chances of heterokaryon formation. On the one hand, this phenomenon is advantageous, since it leads to efficient blocking of virus transfer, that is, limits the spread of harmful cytoplasmic or nuclear elements (Caten, 1972), protection of a local population from 'invader', and prevent resource plundering (Debets and Griffiths, 1998). On the other hand, this incompatibility blocks the transfer of genetic elements between different isolates; this is visualized as the inability to form heterokaryon on selected media (van Diepeningen et al., 1997). However, the formation of heterokaryons between different strains is a common and important aspect of the life cycle of many filamentous fungi (de Carvalho and Mendes-Costa, 2011). Thus the high degree of incompatibility raise the question of how is recombination possible between different isolates when both the sexual and parasexual cycles are missing or blocked (Pal, 2007). It was on this basis, we decided to check the variability in isolates from the various food substance and their vegetative compatibility levels, perhaps, and this might reveal some cryptic sexual or parasexual cycle. In several fungi, vegetative compatibility groups have been correlated with race, enzyme, nucleic acid, polymorphisms, host range, secondary metabolite production, pathogenicity and morphology (Goh et al., 2014; Merzoug and Belabid 2017; Horn et al., 1996). Thus, we induced mutation in our isolates, and based on the information generated, vegetative compatibility groups were established. This was use in this research to demonstrate the extent of genetic diversity among 
A. niger isolated from various food substances in Benin City, Nigeria

\section{MATERIALS AND METHODS}

A. niger strains: Aspergillus niger strains were isolated from eight food substances in the Department of Plant Biology and Biotechnology, University of Benin, Benin City, Edo state, Nigeria. The strains were AspnM, AspnP, AspnZ, AspnO, AspnG, AspnGS, AspnY and AspnB and all these strains were identified as A. niger on Potato Dextrose Agar (PDA) using morphological characteristics by Samson et al., 2007.

Mutagenesis of Aspergillus niger: The mutagenesis follows the protocol described by Scriban (1988). Spores of Aspergillus niger were put in a test tube that contained $100 \mathrm{~mL}$ of acetate buffer $(\mathrm{pH}=4.0)$. Thereafter, $0.5 \mathrm{~mL}$ of sodium nitrite $(0.2 \mathrm{~g} / \mathrm{L})$ was added and the mixture was incubated in bainmarie at $30^{\circ} \mathrm{C}$ for $1 \mathrm{~h}$. To stop the reaction, $200 \mathrm{~mL}$ of phosphate buffer $(\mathrm{pH}=7.0)$ was added to the content of the tube placed in melting ice. At $\mathrm{pH}$ lower than 6.0, all nitrites formed from the nitrous acid which is a strong chemical mutagen that was needed. The spores were taken out of the solution and cultivated on PDA culture medium at the rate of $100 \mu \mathrm{L}$ per petri dish. The cultures were incubated for $30^{\circ} \mathrm{C}$ until it was matured. From the morphological characteristics of colonies, the mutants were localized.

Media: In this work, Potato Dextrose Agar (PDA) was routinely used as complete medium; Basal medium (BM) was prepared as follows, per litre of distilled water: $30 \mathrm{~g}$ of sucrose, $1 \mathrm{~g}$ of $\mathrm{KH}_{2} \mathrm{PO}_{4}, 0.5 \mathrm{~g}$ of $\mathrm{MgSO}_{4} \cdot 7 \mathrm{HO}, 0.5 \mathrm{~g}$ of $\mathrm{KCl}, 10 \mathrm{~g}$ of $\mathrm{FeSO}_{4} \cdot 7 \mathrm{HO}, 20 \mathrm{~g}$ of agar and $0.2 \mathrm{ml}$ of trace element solution following Solomonson and Vennesland 1972; Chlorate resistant sectors (CRSs) were generated on potato dextrose agar (PDA) containing $2.5 \%$ chlorate $\left(\mathrm{KClO}_{3}\right)$, represented as PDC; Minimal medium (MM) was prepared by adding $2 \mathrm{~g}$ of sodium nitrate $\left(\mathrm{NaNO}_{3}\right)$ to $1 \mathrm{~L}$ of basal medium following Correll et al., (1987).

Vegetative Compatibility Groups (VCGs): Vegetative compatibility tests were carried out by making all possible pairings in twos on potato dextrose agar (complete medium) plates and there were 60 possible combinations consisting of both the wild type and mutant isolates of Aspergillus niger. A key was then generated of which '+' represents compatible and '.' represents incompatible.

Generation of Nit Mutant: Fragments of mycelium for each strain were transferred from CM to PDA containing $2.5 \% \mathrm{KClO}_{3}$, presented as PDC. The plates were incubated for 14 days and the chlorate resistant sectors were transferred to MM afterwards. $2 \mathrm{~mm}^{2}$ of a mycelium fragment of the nit mutants were sub cultured on each of the three media containing different nitrogen sources; $\mathrm{NO}_{3}{ }^{-}, \mathrm{NO}_{2}{ }^{-}$and $\mathrm{NH}_{4}{ }^{+}$. The mutants were transferred to each phenotyping medium and were incubated in room temperature for 7 days. The nit mutants were classified into four phenotypic classes (nit 1, nit 3, nit $\mathrm{M}$ and crn) according to Correll et al., 1987.

Complementation Test: Nit mutants from the same isolates were paired each on minimal media plate in all possible combinations (Nit $\mathrm{M} *$ nit 1 , nit $1 *$ nit3, nit3

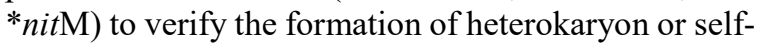
compatible (HSC) isolate. The pairings were incubated in a completely dark chamber and complementation was scored after 7 to 14 days of culturing.

\section{RESULTS AND DISCUSSION}

In order to estimate the variability inherent in the $A$. niger isolated from various host, the sizes of sporangia of the wild and mutants were measured under the same condition. Following the results, the sporangia sizes exhibited higher level of variability when the mutants were matched against the wild types rather than wild versus wild or mutant versus mutant. However, using unpaired-t test, there was significant difference between the mutants and wild of the sporangia. The variation in the sporangia size is as shown in fig. 1 and fig.2. The results inferred that $\mathrm{AspnB}_{1}$ had the largest size of sporangium while AspnP $\mathrm{P}_{1}$ had the smallest size of sporangium (in the mutants). While in the wild, AspnM and AspnB had the largest and smallest size of sporangium respectively.

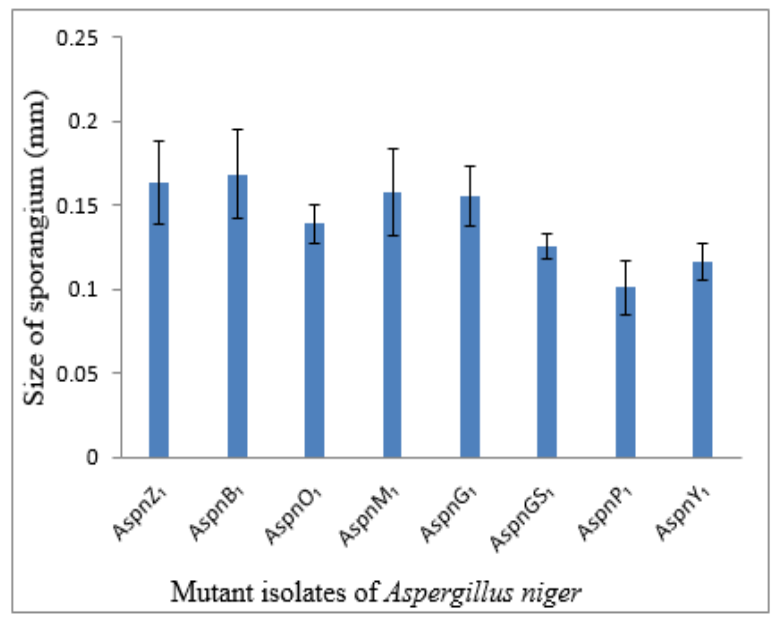

Fig 1: Variation in sporangium size of the mutant isolates of Aspergillus niger. Aspn $\mathrm{B}_{1}$ had the largest size of sporangium while AspnP $_{1}$ had the smallest size of sporangium (in the mutants) when viewed under the light microscope at a magnification of $\times 400$. 


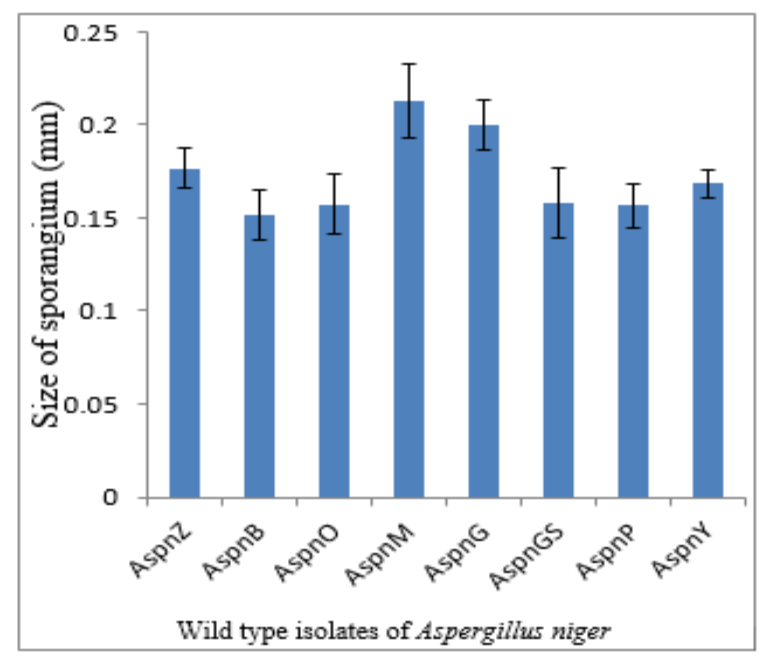

Fig 2: Variation in sporangium size of the wild type isolates of $A$. niger AspnM and AspnB (in the wild) had the largest and smallest size of sporangium respectively when viewed under a light microscope at a magnification of $\times 400$

Since we maintained the same growth condition throughout the experiment, our focus was on inherent differences that could aid genotyping. Based on the variation observed in the sporangia, pairing of the different strains was carried out in all possible form, using the mutant strains. This was done in order to determine the compatibility levels. As a result of the pairing, four classes of vegetative compatibility groups (VCGs) were generated (Table1). The number of VCGs obtained from the mutant strains of $A$. niger include; VCG-1 (AspnB ${ }_{1}, A^{A s p n Y_{1}}$ and $\left.A_{\text {spn }}{ }_{1}\right)$, VCG$2\left(\text { AspnO }_{1}, \text { AspnGS }_{1} \text { and AspnZ }\right)_{1}, V_{C G} 3$ (AspnG $_{1}$, $\left.\mathrm{AspnO}_{1}, \mathrm{AspnZ}_{1}\right)$ and $\mathrm{VCG}-4\left(\mathrm{AspnM}_{1}, \mathrm{AspnG}_{1}\right.$ and $\mathrm{AspnO}_{1}$ ) which contain three isolates in each group. Having determined the various compatibility groups, complementation test quickly followed. This was carried out using minimum medium. Complementation in this medium ought to have led to formation of heterokaryon (i.e., the mutants being paired generating the wild type at midpoint where the mutants strains met). Interestingly, the results shows that there is no heterkaryon formation in any of the pairing between vegetative compatibility groups, thus confirming the distinctness of each group genetically. The initial process of heterkaryon formation involves hypha fusion which is controlled genetically by heterokaron incompatibility (het) gene (Glass and Kuidau, 1992) or vegetative incompatibility (vic) gene (Leslie 1993). In this research, we observed that there was no heterokaryon formation among the $A$. niger isolated from the various food substances.

Table 1: Vegetative compatibility test on the mutant isolates of Aspergillus niger

\begin{tabular}{|c|c|c|c|c|c|c|c|c|}
\hline AspnM $_{1}$ & AspnY $_{1}$ & AspnZ & $\mathrm{AspnP}_{1}$ & $\mathrm{AspnG}_{1}$ & AspnGS $_{1}$ & $\mathrm{AspnB}_{1}$ & $\mathrm{AspnO}_{1}$ & \\
\hline \multirow[t]{8}{*}{+} & + & - & - & - & - & - & - & AspnM $_{1}$ \\
\hline & + & - & + & + & + & + & + & AspnY $_{1}$ \\
\hline & & + & + & + & + & + & + & AspnZ $_{1}$ \\
\hline & & & + & - & + & + & + & AspnP $_{1}$ \\
\hline & & & & + & - & - & + & AspnG $_{1}$ \\
\hline & & & & & + & - & + & AspnGS $_{1}$ \\
\hline & & & & & & + & - & AspnB $_{1}$ \\
\hline & & & & & & & + & AspnO $_{1}$ \\
\hline
\end{tabular}

Key: +: Compatible; -: Incompatible.

This suggests that genetically different individual cannot exchange genetic material in this fungus. However, this statement does not mean $A$. niger forbids their mycelia from lying side by side or even fusing. In an attempt to circumvent the genetic recombination obstacles, we decided to fuse the different isolates in the wild form knowing that the mycelia will anastomose and form vegetative group but with the inability to exchange genetic material will degenerate. Also, mutants of the A. niger were created and pairing of the various mutants followed. The pairing resulted to classification of the various isolates into different vegetative compatibility groups. The compatibility measure in this work was the absence of barrage formation between pairs, this is so, due to the obvious absence of heterokaryon formation. Barrage formation is one of the ways established scientifically for different isolates that are not compatible (Leslie,
1993). If strains are different but genetically compatible, they are supposed to fuse and result in heterokaryon formation on a selective medium as seen in many other filamentous fungi. This is demonstrated by the two pairing mutants that resulted in the wild type at the midpoint where their hypha fused due to gene complementation by the presence of two different nuclei in one hypha. However, in A. niger, we observed that, cases where mutants are of the same vegetative compatibility group, their mycelia meet without any barrage formation on minimum medium (fig3) but with the absence of heterokaryon formation. This suggests no exchange of nuclear or genetic material. It also suggests that the mycelia can only relate vegetatively in $A$. niger. 


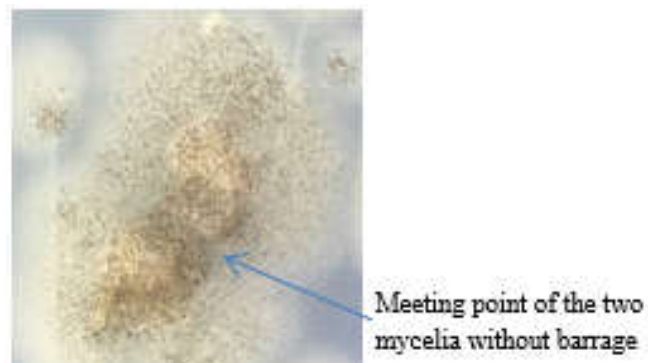

Fig 3. Nit-1 AspnY and NitMAspnB mutants paired on MM

Our judgment of differences in isolates based on vegetative compatibility groups is clearly defined, however the presence of some strains in more than one vegetative compatibility group, suggest polymorphism in the het loci. Saupe (2000) stated that the function of incompatibility loci in fungi is not clear though it sometimes refer to the reaction between genetically different individuals. We attempted to highlight the differences by creating mutants of the wild followed by comparism of their sporangia sizes. It was interesting to note that there was significant difference in the sporangia. Since there was a significant difference in the sizes of the sporangia, it was therefore logical to expect a difference in the number of conidia per sporangium.

This work suggests that, there are four genotypes of $A$. niger based on the VCGs. However, the heavy production of melanin by this fungus could limit their differences, since melanin could resist mutational changes due to ultra violet light or other mutagenic substances.

Conclusion: From this experiment, there is no heterokaryon formation in $A$. niger. The reason for the none formation of heterokaryon could be that $A$. niger prefer to maintain its individuality. In agreement with other researchers, heterokaryon incompatibility genes exist to limit heterokaryon formation between unlike individuals. This means that the het genes in A. niger is there to preserve genetic individuality. Based on this, it is safe to classify different strains as genotypes in $A$. niger using vegetative compatibility group.

\section{REFERENCES}

Caten, CE (1972). Vegetative incompatibility and cytoplasmic infection in fungi. J. Gen. Microbiol. 72(2): 221-229.

Correll, JC; Klittich, CJR; Leslie, JF (1987). Nitrate non utilizing mutants of Fusarium oxysporum and their use in vegetative compatibility test. Phytopathology, 77: 1640- 1646.
Pal, K (2007). Comparative Analysis of Genetic Incompatibility in Aspergillus niger and Podospora Anserina, Ph.D-Thesis Wageningen University, 135pp.

de Carvalho, CR; Mendes-Costa, MC (2011). Vegetative compatibility and heterokaryon formation between different isolates of Colletotrichum lindemuthianum by using the nit mutant system. Braz. J. Microbiol. 42(1): $346-$ 353.

Debets, AJM; Griffiths AJF (1998). Polymorphism of het-genes prevents resources plundering in Neurospora crassa Mycol Res. 102:1343-1349.

Diener, UL (1960). The mycoflora of peanuts in storage. Phytopatholology, 50: 220 - 223.

Frisvad, JC; Peterson, LM; Lyhne, EK; Larsen, TO (2014). Formation of sclerotia and production of indoloterpenes by Aspergillus niger and other species in section Nigri. PLOS. 9(4): $1-11$.

Gautam, AK; Sharma, S; Avasthi, S; Bhadauria, R (2011). Diversity and toxicology of A. niger: an important spoilage fungi. Res. J. Microbiol. 6(3): $270-280$.

Glass, NL; Kuldau, GA (1992). Mating-type and Vegetative incompatibility in filamentous ascomycetes Anau. Rev. Phytopathol. 30:201-224

Goh, KM; Ganeson, M; Supramaniam, CV (2014). Infection potential of vegetative incompatible Ganoderma bonisense isolates with known ligninolytic enzyme production. Afri. J. Biotechnol.13(9): 1056 - 1066.

Horn, BW; Greene, RL; Sobolev, VS; Dorner, JW; Powell, JH (1996). Association of morphology and mycotoxin production with vegetative compatibility groups in Aspergillus flavus, $A$. parasiticus and A. tamari. Mycologia, 88: 574587

Legisa, M; Mattey, M (2007). Changes in primary metabolism leading to citric acid overflow in Aspergillus niger. Biotechnol. Lett. 29: 181-190

Merzoug, A; Belabid, L (2017). Relationship between pathogenicity, race and vegetative compatibility grouping among Algerian populations of Fusarium oxysporum $F$. sp. pisi causing pea wilt. J. Plant Prot. Res. 57(4):370-378 
Papagianni, M (2007). Advances in citric acid fermentation by Aspergillus niger: biochemical aspects, membrane transport and remodeling. Biotechnol. Adv. 25(3): 244-263

Samson, RA; Noonim, P; Meijer, M; Houbraken, J; Frisvad, JC; Varga, J (2007). Diagnostic tools to identify black Aspergilli. Stud.Mycol. 59: 129 145.
Scriban, R (1988). Biotechnologie, Technique et Documentation. Lavosier, 3. Auflage, Paris, 906 Seiten, mit zahlreichen Abbildungen und Tabella. 41(6):245-245

Show, PL; Oladele, KO; Siew, QY; Zakry, FAA; Lan, JCW; Ling, TC (2015). Overview of citric acid production from Aspergillus niger. Front Life Sci. 8(3): $271-283$

Solomonson, LP; Vennesland, B (1972). Nitrate reductase and chlorate toxicity in Chlorella vulgaris Beijerinck. Plant Physiol. 50: 421 - 424. 